\title{
Prevalencia de dengue en pacientes sintomáticos que acuden al Instituto Nacional de Higiene "Leopoldo Izquieta Pérez" de la ciudad de Puyo (primer semestre del 2010)
}

\author{
ANDREA PAULINA VELASTEGUÍ SÁNCHEZ ${ }^{*}$ * ÁNGEL HERNÁN TOBAR SALTOS², \\ EDGAR VICENTE MORA BRITO3${ }^{3}$, WILFRIDO TORRES ALVARADO ${ }^{4}$, ISABEL FIERRO AGUAS \\ 'Facultad de Ciencias Químicas-Universidad Central del Ecuador. Ciudadela Universitaria y Gatto Sobral. \\ ${ }^{2} \mathrm{NHH}$ LIP Pastaza. ${ }^{3}$ Hospital Provincial Puyo. ${ }^{4}$ SNEM Pastaza. \\ *Correspondencia: pauly_2112@hotmail.com.
}

Recibido: 09 marzo 2012 / Aceptado: 18 octubre 2012

\section{Resumen}

El objetivo del presente estudio fue determinar la prevalencia del dengue en pacientes sintomáticos que acudieron al Instituto Nacional de Higiene "Leopoldo Izquieta Pérez" de la ciudad de Puyo y su relación con las condiciones climáticas en el período comprendido entre enero y mayo de 2010, a través de un estudio transversal-descriptivo. Durante los meses de estudio, acudieron un total de 490 pacientes provenientes de diversas localidades, se registró información de datos demográficos tales como: el género, edad, lugar de residencia por barrios de la ciudad de Puyo y de otras localidades. El diagnóstico serológico de laboratorio se realizó mediante la técnica de micro ELISA, en donde se obtuvieron 326 casos positivos para anticuerpos lgM y 164 negativos. Se calculó la prevalencia de dengue de acuerdo a los resultados obtenidos, siendo del $66,5 \%$ con un intervalo de confianza al 95\% [62,1\% - 70,7\%]. El estudio demostró que las condiciones climáticas no son estadísticamente significativas $(P>0,05)$ como determinantes para el aumento de casos de dengue, sugiriendo más bien que éstos pueden estar relacionados a otros factores. El $84,6 \%$ de los casos positivos fueron habitantes de la ciudad de Puyo, de los cuales el 44,2\% se concentró en los barrios: "Obrero", "Ciudadela del Chofer" y "Vicentino", zonas geográficamente ubicadas en el norte de la ciudad y aledañas entre sí. Concluimos que la infección por dengue en la ciudad de Puyo constituye un problema emergente que debe ser atendido oportunamente por las autoridades de salud.

Palabras clave: Dengue, anticuerpos $\lg G$ e $\lg M$.

\section{Prevalence of dengue in sintomatics patients who come to the National Institute of Hygiene "Leopoldo Izquieta Pérez" of the city of Puyo, (first half of 2010)}

\section{Abstract}

The objective of this study was to determine the prevalence of dengue fever in symptomatic patients who went to the National Institute of Hygiene "Leopoldo Izquieta Pérez" of the city of Puyo and its relationship with the climatic conditions in the period between January and May of 2010, through a cross-sectional study-descriptive. During the months of study, went a total of 490 patients from various locations, was recorded information of demographic data such as: gender, age, place of residence by neighborhoods of the city of Puyo and other locations. The serological diagnosis of laboratory was carried out through the technique of micro ELISA, where he obtained 326 positive cases for IgM antibodies and 164 negative. It is estimated the prevalence of dengue according to the results obtained, being of 66.5 percent with a range of confidence in the 95 per cent $[62.1 \%$ $70.7 \%]$. The study showed that the climatic conditions are not statistically significant ( $P>0.05)$ as crucial to the increase in cases of dengue fever, but that may be linked to other factors. The 84.6 percent of positive cases were inhabitants of the city of Puyo, of which the $44.2 \%$ concentrated in the neighborhoods "Obrero", Ciudadela del Chofer" and "Vicentino", neighborhoods geographically located in the North of the city and are surrounding 
among themselves. We conclude that the infection by dengue in the city of Puyo constitutes an emerging problem that needs to be addressed appropriately by health authorities.

Key words: Dengue, $\lg G$ and $\lg M$ antibodies.

\section{Introducción}

En el año 2010, la epidemia nacional de dengue, involucró a la ciudad de Puyo, convirtiéndose en un problema local de salud pública que merece ser estudiado lo suficiente para establecer el comportamiento del brote y aportar con información real que permita mejorar el abordaje hacia el control de la enfermedad.

El dengue es la enfermedad ocasionada por el arbovirus con mayor morbilidad y mortalidad en el mundo. Aproximadamente, la mitad de la población mundial vive en países donde es endémico y se estima que anualmente ocurren cerca de 50 millones de casos de dengue, los que originan aproximadamente 24.000 muertes. [1]

En la mayoría de los países, la transmisión de la enfermedad y la frecuencia de sus epidemias ha aumentado, debido al rápido crecimiento de las poblaciones urbanas con malos servicios de saneamiento, falta de recursos adecuados para el almacenamiento de agua y desechos de basura, condiciones óptimas para la propagación del Aedes aegypti. [2]

El aumento del número de casos ocurre durante la estación de lluvia dado el incremento de la población del Aedes aegypti. Los pacientes son generalmente jóvenes y niños/as, debido a que la mayoría de adultos/as ya ha sufrido la enfermedad y han desarrollado inmunidad contra el serotipo presente.

El dengue es un problema creciente para la salud pública mundial debido a varios factores: el cambio climático, el aumento de la población mundial en áreas urbanas de ocurrencia rápida y desorganizada, la insuficiente provisión de agua potable que obliga a su almacenamiento en recipientes caseros habitualmente descubiertos, la inadecuada recolección de residuos y la gran producción de recipientes descartables que sirven como criaderos de mosquitos, al igual que los neumáticos desechados. A esto se suman: el aumento de viajes y migraciones, las fallas en el control de los vectores, y la falta de una vacuna eficaz para prevenir la enfermedad. [2]

Por ello, el objetivo de esta investigación, fue determinar la prevalencia de dengue en pacientes sintomáticos que acuden al Instituto Nacional de Higiene "Leopoldo Izquieta Pérez", de la ciudad de Puyo, cantón Pastaza.

\section{Parte experimental}

El estudio se realizó con todas aquellas personas que acudieron al Instituto Nacional de Higiene "Leopoldo Izquieta Pérez" de la ciudad de Puyo, durante los meses de enero a mayo de 2010. Con los pacientes que se presentaron durante este tiempo, se procedió a cumplir con ciertos criterios previos a la obtención de la muestra de sangre, que incluyeron: datos informativos del paciente (nombres completos, edad, sexo), lugar de residencia (zona urbana o rural), domicilio exacto (calles y teléfono, para sectorizar los focos infecciosos en la ciudad), signos y síntomas actuales (días febriles, temperatura, náuseas, dolor retro ocular, muscular, cefalea, letargia, gingivorragia, petequias), luego de dicha recolección, se procedió a la toma de muestra.

Se incluyó en el estudio a todos los/as pacientes con síntomas febriles, de cualquier edad y sexo, que acudieron con un previo diagnóstico clínico (evaluados por un médico al Instituto) para realizarse la prueba confirmatoria serológica de dengue. La prevalencia de la enfermedad se estimó de acuerdo a los resultados de la prueba de diagnóstico de anticuerpos lgG e $\lg M$, con un intervalo de confianza de $95 \%$.

Cada muestra de suero se analizó siguiendo el procedimiento, normas y estándares propios de la técnica de microelisa. La variable del estudio fue detectar la presencia-ausencia de anticuerpos IgM contra el virus del dengue. Los sueros humanos investigados se consideraron positivos, si el título de anticuerpos lgM superaba el valor de corte (Cut off) calculado, con más 
de $1 \mathrm{U} / \mathrm{ml}$ de concentración de anticuerpos, debido a que estos anticuerpos $\lg M$, expresan infección reciente mientras que los lgG, son anticuerpos de memoria.

Para determinar la relación entre condiciones climáticas-aumento de casos de dengue, se realizó un análisis estadístico utilizando una prueba paramétrica t de Student, con una prueba de Levene para la igualdad de varianzas y prueba $\dagger$ para la igualdad de medias, buscando establecer si son o no significativas.

Adicionalmente, se realizó una comparación de títulos de anticuerpos de memoria en pacientes positivos y negativos, utilizando un análisis estadístico para muestras independientes con comparación de medias.

Todas las pruebas estadísticas fueron interpretadas de acuerdo al valor $\mathrm{P}$ al $95 \%$ de intervalo de confianza (I.C.). Para el análisis de los datos se utilizaron los paquetes estadísticos SPSS $₫$ versión 1.8 y Epi Info $\AA$ versión 3.3.2 para Windows.

\section{Resultados y análisis}

De las 490 muestras analizadas, se encontraron un total de 326 muestras positivas de acuerdo al siguiente criterio: se consideró como muestra positiva si el título de anticuerpos lgM superaba el valor de corte (Cut off) calculado, con más de $1 \mathrm{U} / \mathrm{ml}$ de concentración de anticuerpos, debido a que los anticuerpos $\lg M$, expresan infección reciente mientras que los $\lg G$, son anticuerpos de memoria.

\subsection{Cálculo de la prevalencia}

La prevalencia de dengue se calculó en base a los resultados obtenidos de la prueba de diagnóstico por microELISA de dengue, realizada a pacientes sintomáticos que acudieron al Instituto Nacional de Higiene "Leopoldo Izquieta Pérez", de la ciudad de Puyo, durante el periodo de enero a mayo de 2010 , siendo del $66,5 \%$ con un intervalo de confianza al $95 \%$ de $[62,1 \%-70,7 \%]$ Tabla 1.

Tabla 1. Prevalencia de dengue total

\begin{tabular}{ccccc}
\hline $\begin{array}{c}\text { Diagnóstico Infección } \\
\text { Dengue IgM }\end{array}$ & Frecuencia & Porcentaje & $\begin{array}{c}\text { Porcentaje } \\
\text { acumulado }\end{array}$ & I.C. 95\% \\
\hline Negativo & 164 & $33,5 \%$ & $33,5 \%$ & $29,3 \%-37,9 \%$ \\
Positivo & 326 & $66,5 \%$ & $100,0 \%$ & $62,1 \%-70,7 \%$ \\
Total & 490 & $100,0 \%$ & $100,0 \%$ & \\
\hline IC: Indice de confianza al 95\% & & & & \\
ELABORACIÓN: Paulina Velasteguí, & & &
\end{tabular}

De acuerdo a los registros de precipitación, temperatura y humedad del INAMHI, zona Norte-Pastaza y los casos positivos de dengue diagnosticados en el INH durante el estudio, se realizó un análisis estadístico utilizando una prueba paramétrica t de Student, con una prueba de Levene para la igualdad de varianzas y prueba † para la igualdad de medias, con una significancia de P>0,05. Tabla 2.

Tabla 2. Tabla de relación entre prevalencia de dengue y condiciones climáticas

\begin{tabular}{|c|c|c|c|c|}
\hline $\begin{array}{l}\text { Parámetros } \\
\text { climáticos }\end{array}$ & $\begin{array}{r}\text { Diagnósti } \\
\text { Infección de }\end{array}$ & $\begin{array}{l}\text { IgM } \\
\text { lengue }\end{array}$ & Media $( \pm D E)$ & p-Valor \\
\hline \multirow{2}{*}{ PRECIPITACION MEDIA (mm) } & \multirow{2}{*}{ dimension1 } & Positivo & $393,357( \pm 76,45)$ & \multirow{2}{*}{$0,180^{*}$} \\
\hline & & Negativo & $383,601 \pm(74,83)$ & \\
\hline \multirow{2}{*}{ TEMPERATURA MEDIA $\left({ }^{\circ} \mathrm{C}\right)$} & \multirow{2}{*}{ dimension1 } & Positivo & $23,168 \pm(0,27)$ & \multirow{2}{*}{$0,145^{\star}$} \\
\hline & & Negativo & $23,206 \pm(0,26)$ & \\
\hline \multirow{2}{*}{$\%$ HUMEDAD MEDIA (\%) } & \multirow{2}{*}{ dimension1 } & Positivo & $86,61 \pm(0,89)$ & \multirow{2}{*}{$0,125^{\star}$} \\
\hline & & Negativo & $86,49 \pm(0,83)$ & \\
\hline
\end{tabular}


De esta forma, no se encontró diferencia estadística significativa $(P>0,05)$ en relación a las condiciones climáticas con el aumento de prevalencia de dengue, por lo tanto, se rechaza la hipótesis que sostiene que: "Existe una mayor prevalencia de dengue relacionada a las condiciones climáticas en la época invernal en los pacientes sintomáticos que acuden al Instituto Nacional de Higiene "Leopoldo Izquieta Pérez" de la ciudad de Puyo, cantón Pastaza"; por lo que, las poblaciones son las mismas y las condiciones climáticas no son determinantes para el aparecimiento de casos de dengue.

\subsection{Análisis de los valores de anticuerpos lgG en los pa- cientes que acudieron al INHLIP durante el estudio}

De los títulos de anticuerpos $\lg G$ obtenidos en pacientes positivos y negativos, se encontró que la respuesta de anticuerpos de memoria en pacientes positivos es el doble que la que tuvieron los pacientes negativos para infección reciente de dengue, de acuerdo a un análisis estadístico para muestras independientes con comparación de medias en donde se obtuvo una significancia de $\mathrm{P}<0,05$. Tabla 3.

Tabla 3. Valores de $\lg G$ en los pacientes analizados

\begin{tabular}{ccccc}
\hline & $\begin{array}{c}\text { Pacientes } \\
\text { analizados (IgM) }\end{array}$ & $\mathbf{N}$ & $\begin{array}{c}\text { Anticuerpos IgG } \\
\text { Media }( \pm \mathrm{DE})\end{array}$ & p-Valor \\
\hline IgG & Positivos & 326 & $2,226( \pm 1,50)$ & $0.00^{*}$ \\
& Negativos & 164 & $1,181( \pm 1,38)$ & $0.00^{*}$ \\
& TOTAL & 490 & & \\
\hline
\end{tabular}

$\mathrm{N}^{\circ}$ casos dengue positivo $=326$

$\mathrm{N}^{\circ}$ casos dengue negativo $=164$

${ }^{*} p<0,05$

De esta forma, se determinó que existe diferencias estadísticas altamente significativas $(P<0,05)$ entre los títulos de anticuerpos $\lg G$ de los pacientes que resultaron positivos a la prueba de infección reciente de dengue $(\lg M)$, y los anticuerpos de los pacientes negativos, en donde, los pacientes positivos manifestaron altos títulos de anticuerpos lg $G$ en relación a los negativos, indicando que la exposición posterior al virus del dengue, da lugar a la activación de linfocitos $B$ de memoria y a una respuesta humoral más intensa y rápida. [3]

\section{Discusión}

Durante el año 2010, el dengue se ha presentado como una enfermedad muy conocida en la localidad y que fue padecida por un alto porcentaje de la pobla- ción superando altamente el número de casos reportados en años anteriores.

Se determinó una prevalencia del $66,5 \%$ con un I.C. $95 \%[62,1 \%-70,7 \%]$, en pacientes sintomáticos que acudieron al INHLIP durante el periodo enero-mayo de 2010, utilizando una prueba de diagnóstico de micro ELISA para anticuerpos $\lg G$ e $\lg M$. La prevalencia está relacionada a los 326 pacientes que resultaron positivos para anticuerpos $\lg M$ de un total de 490 individuos.

El porcentaje de prevalencia encontrado es alto, destacando que este resultado, es un valor de los casos confirmados por el INHLIP durante el estudio, pero no significa la prevalencia en general de la provincia de Pastaza, debido a que no todas las personas infectadas con dengue acudieron al mismo para realizarse la prueba confirmatoria durante este período.

Debemos considerar que la prevalencia del virus en el Ecuador es de $75 \%$, lo cual representa a 10.253 casos de dengue padecidos en el país, según datos del Ministerio de Salud. Esta es la razón por la cual constituye un problema emergente de salud pública. [4]

Se encontró que las condiciones climáticas durante los meses de enero-mayo de 2010 no varían. Respecto a los valores de temperatura y humedad, éstos permanecen constantes, mientras que en lo que se refiere a la pluviosidad, las lluvias se incrementan notablemente a partir del mes de abril y mayo [5]. El análisis estadístico entre las condiciones climáticas y la prevalencia de dengue no es significativo $(P>0,05)$, por lo que aquellas, no son un determinante para la aparición de casos de dengue, enfermedad que puede estar más bien relacionada a otros factores: geográficos, culturales, de saneamiento, de abastecimiento o almacenamiento inadecuado de agua potable, migraciones y turismo en la provincia. [6] Se debe considerar también que la región y la provincia poseen dos estaciones climáticas permanentes: húmeda y semi húmeda, por lo que existen lluvias durante todo el año, y el Aedes aegypti, vector del dengue, puede reproducirse en cualquier momento.

En cuanto a la relación de anticuerpos de memoria en pacientes positivos y negativos, la respuesta de anticuerpos $\lg G$ en los pacientes positivos fue altamente superior a la de los negativos. Esto tiene una explicación 
inmunológica ya que las respuestas de anticuerpos a una exposición primaria se deben a la activación de linfocitos $B$ vírgenes que expresan $\lg M$ no estimulados previamente, mientras que las secundarias se deben a la estimulación de clones expandidos de linfocitos $B$ de memoria que expresan lgG. Por lo tanto, la respuesta secundaria aparece más rápidamente que la respuesta primaria, y en la respuesta secundaria, se sintetizan cantidades mayores de anticuerpos. [3]

De esta forma, se encontró que existieron pacientes que expresaron anticuerpos de memoria lgG, lo cual significa que estos individuos ya estuvieron alguna vez expuestos al virus del dengue y desarrollaron anticuerpos, lo cual probablemente pasó como una infección inadvertida y asintomática; por lo tanto, estos pacientes constituyen una población en riesgo de padecer dengue hemorrágico en posteriores ocasiones.

\section{Conclusiones}

- La prevalencia de dengue en pacientes sintomáticos que acudieron al Instituto Nacional de Higiene
"Leopoldo Izquieta Pérez", de la ciudad de Puyo, durante el periodo enero-mayo del 2010, fue del $66,5 \%$.

- El dengue es la arbovirosis con mayor morbilidad y mortalidad en el mundo convirtiéndose en un problema creciente para la salud pública nacional y mundial.

- Las condiciones climáticas no fueron un determinante para el aumento de prevalencia de dengue en los pacientes sintomáticos que acudieron al INHLIP durante el estudio.

- La respuesta de anticuerpos de memoria en pacientes positivos es superior a la respuesta de los negativos debido a previas exposiciones al virus del dengue por parte de estos pacientes.

- En el año 2010, el dengue afectó a gran parte de la población, convirtiéndose en una enfermedad emergente para las autoridades de salud en la provincia.

\section{Referencias bobliográficas}

1 GUHA, S., Schimmer, B. (2005) Dengue fever: new paradigms for a changing epidemiology. Emerg Themes Epidemiol; 2: 1

2 FERNANDEZ, R. (2004) Medicina Tropical. Patologías Tropicales: aspectos científicos, sociales y preventivos. Tercera Edición. GuayaquilEcuador.

3 ABBAS, A. (2009) Inmunología celular y molecular. Sexta Edición. Editorial Elsevier. Capítulo 10. Activación de los linfocitos B y producción de anticuerpos. Pág. 215-218. Capítulo 14. Mecanismos efectores de la inmunidad humoral. Pág. 321-323

4 Ministerio de Salud Pública. (2007). Boletín para el manejo de emergencia de dengue en el Ecuador

5 INAMHI (2010). Instituto Nacional de Meteorología e Hidrología. Estación Meteorológica Puyo-Pastaza. http://www.inamhi.gov.ec

6 NELSON, M. J. (2006). Aedes aegypti: Biología y Ecología. Organización Panamericana de la Salud. REF: PNSP/86-93. Washington, D.C: 50 